\title{
Post-covid Rhino Cerebral Mucor Mycosis: Neurosurgical Experience
}

\section{Samrendra Kumar Singh ${ }^{1}$, Brajesh Kumar ${ }^{2 *}$ Niraj Kanaujia $^{2}$ Om Prakash Gupta ${ }^{2}$, KM Jha ${ }^{3}$ and Rakesh Kumar Singh ${ }^{4}$}

${ }^{1}$ Associate Professor, Department of Neurosurgery, IGIMS, Patna, India

${ }^{2}$ Assistant Professor, Department of Neurosurgery, IGIMS, Patna, India

${ }^{3}$ Professor and Head of Department, Department of Neurosurgery, IGIMS, Patna,

India

${ }^{4}$ Professor and Head of Department, Department of Otorhinolaryngology, IGIMS,

Patna, India

*Corresponding Author: Brajesh Kumar, Assistant Professor, Department of

Neurosurgery, IGIMS, Sheikhupura, Raza bazar, Patna, Bihar, India.

E-mail: drbrajesh78@gmail.com
Received: September 22, 2021

Published: November 19, 2021

(C) All rights are reserved by Brajesh Kumar., et al.

\begin{abstract}
Objective: Mucor-mycosis is a fungal infection which usually develops in severe immunocompromised patients, e.g., post-transplant, uncontrolled Diabetes Mellitus, malignancies, and iatrogenic immunosuppression. We faced flood of post-Covid Rhino cerebral Mucor mycosis cases at our institution after second wave of Covid from April to July 2021. We present our experience of ten patients who underwent neurosurgical intervention.

Patients and Methods: This was a prospective study of ten patients who underwent neurosurgical intervention for Rhino cerebral Mucor mycosis. All cases were operated by same surgeons from ENT and Neurosurgery. Disseminated brain abscess, multilobe involvement, brainstem involvement and isolated cavernous sinus thrombosis excluded from this study.

Results: Total forty-five patients referred for neurosurgical opinion, out of those ten patients taken for neurosurgical intervention. Out of ten, nine patients had history of Covid infection (RT-PCR positive). They were treated with steroid and oxygen inhalation. One patient had history of fever, but his Covid RT-PCR was negative. He was treated at home with oxygen. All patients were treated with either liposomal amphotericin B or conventional Amphotericin B depending on the availability and affordability of the patient. Oral Posaconazole (100 mg), six to eight hourly was given to all patients for 6weeks to 3 months in gradual tapering doses depending on the nasal endoscopic findings in the follow up. Sinus surgery was performed in all ten patients by ENT Surgeons, two underwent orbital exenteration by eye surgeon, eight patients underwent open craniotomy for cerebral infection while in two patients' cerebral exploration done endoscopically. Eight patients survived, while two patients died.

Conclusion: Patients with rhino-cerebral mucormycotic spreading outside the Sino nasal cavity to the base of brain is treatable by early neurosurgical interventions when the abscess cavity is localized to frontal or temporal skull base.

Keywords: Rhino Cerebral; Mucor-mycosis; Post Covid; Immune Suppression
\end{abstract}

\section{Introduction}

Mucor mycosis is an infection caused by Mucorales fungi, within the class zygomycetes [1]. Rhino cerebral Mucor mycosis is the most common type and occurs usually in the immunocompromised patients with diabetic ketoacidosis, neutropenia, urae- mia, burns, chronic corticosteroid therapy, severe malnutrition, post-transplant, haematological malignancies, and iatrogenic immunosuppression [2-4]. Spores enter the nose by inhalation and then spread to the paranasal sinuses and subsequently to the hard palate, orbit, meninges, and brain by direct extension [1,5]. 
The clinical manifestations of rhino cerebral mucormycotic are swelling around nose, orbit and black discolouration due to thrombosis of vessel for which it was named 'black fungi. Other presentations are fever, headache, lethargy, decreased vision and ophthalmoplegia due to cavernous sinus thrombosis. Death may follow in a few days, especially in undiagnosed and severely immunocompromised patients due to dissemination, vascular invasion, thrombosis, ischemia, and infarction.

Post-Covid rhino cerebral mucormycotic was completely new experience for all of us. There are very few published series of rhino cerebral mucormycotic having enough patients to analyse the predictors of survival in these cases $[1,6,7]$. The purpose of this study is to share our neurosurgical experience in these ten cases.

\section{Patients and Methods}

We prospectively studied the outcomes of patients who underwent neurosurgical intervention for rhino cerebral mucormycotic between June 2021 to July 2021. We analysed the following data for all patients: age, gender, predisposing illness, clinical symptoms and findings, imaging results, pathological diagnosis, microbiological results, laboratory findings, surgical procedures, and treatment outcomes. Magnetic resonance imaging (MRI) scans were done in all the cases.

Biopsies were taken from all patients for the histopathological analysis and culture. In all the cases, histopathological examination identified invasive, broad, thick-walled, non-septate hyphae branching at right angles (Figure 2A and 2B).

All patients received initial empirical treatment with liposomal amphotericin B/Amphotericin B depending on the availability and affordability of the drugs. All patients received Oral Posaconazole (100 mg) six to eight hourly for 6 weeks to 3 months in tapering doses.

\section{Inclusion criteria}

Patients with localized cerebral fungal abscess in the frontal and temporal lobe with or without features of cerebral signs considered for neurosurgical interventions.

\section{Exclusion criteria}

Patients with disseminated cerebral involvement, involvement of cavernous sinuses, cerebral infarct, or ischemia due to thrombosis of blood vessels by fungus were not taken for neurosurgical interventions.

\section{Results}

Total 45 Mucor mycosis patients referred from ENT department for neurosurgical opinion. 19 patients had skull base extension of Mucor mycosis without penetration of durometer were managed medically along with surgical debridement of Paranasal sinuses by ENT surgeon. Among the remaining 26 patients, 6 patients had disseminated cerebral involvement, 6 patients had isolated cavernous sinus involvement and 4 patients had ischemic infarct. These 16 patients were managed medically, and the rest 10 patients were having localized cerebral involvement were selected for neurosurgical intervention.

Out of 10 patients operated, there were 7 males and 3 females, with a mean age of 59.1 years (range 30 to 75 years). Out of 10 patients, 9 were having RT-PCR positive Covid infection one and half months back. They were admitted to hospital and treated with steroids and inhalational oxygen. One patient was RT-PCR negative with history of breathlessness and fever. He was treated with inhalational oxygen without steroids at home. They all recovered from covid infection and discharged from hospital or recovered at home.

Out of 10 patients, 5 were diagnosed case of DM before covid infection and were on oral hypoglycaemics. 2 patients developed uncontrolled DM due to steroid during covid treatment. They were treated with insulin on sliding scale during treatment course in hospital and one patient remained euglycemic.

The common presenting symptoms in all patients were facial oedema, facial pain, and nasal obstruction. On physical examination, findings were nasal discharge, grey or black nasal eschar, periorbital cellulitis, proptosis, ptosis, and altered consciousness (Figure 1).

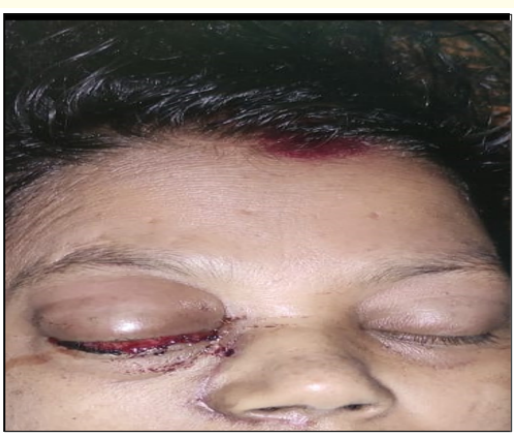

Figure 1: Showing pre-operative image of a patient with periorbital cellulitis, proptosis, and ptosis. 
On initial examination, 6 patients had cutaneous and/or palatal necrosis (one had both palatal and skin necrosis). 2 had ophthalmoplegia and blurring of vision. On neurological examinations 3 patients had one or two episodes of seizure. 2 patients had history of personality changes and 1 patient also had left sided hemiparesis and altered level of consciousness.

On nasal endoscopy, grey or black mucosa was observed on the middle turbinate in all patients. Four patients had large necrotic areas in the nasal cavity. Biopsies were taken from all patients. All patients had a histopathological diagnosis of Mucor mycosis.

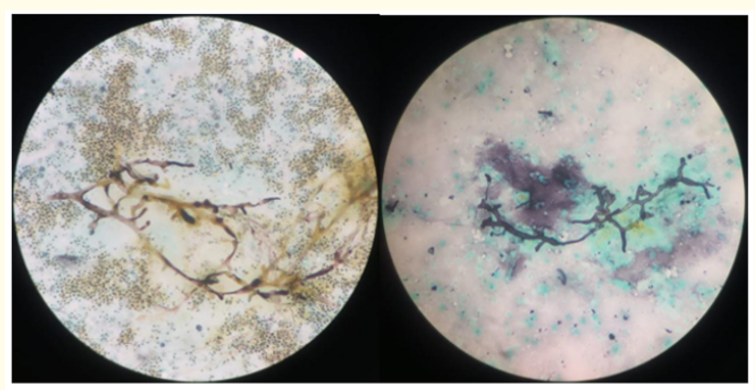

(A)

(B)

Figure 2: A and B: Showing hyphae of Mucor mycosis in microscopy.

Computed tomography (CT) and magnetic resonance imaging (MRI) performed at initial evaluation, showed nonspecific sinusitis findings unilaterally in 8 patients and bilaterally in two. 6 patients had Basi frontal cerebral Mucor mycosis while 4 had Basi temporal Mucor mycosis (Figure 3A and 3B).

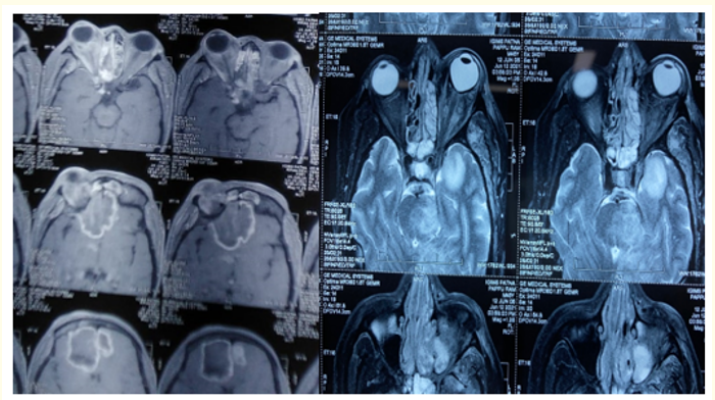

(A)

(B)

Figure 3: A. Showing MRI of two patients with Basi frontal and B. Basi temporal Mucor mycosis.
Patients received a combination of medical and surgical treatment. In Medical management patients received liposomal amphotericin B or plain Amphotericin B depending on the availability and affordability of the patient considering the high cost and long period treatment. All patients were discharged on Oral Posaconazole (100 mg). This oral drug was started 6 - 8 hourly and were tapered depending on the follow up endoscopic examination of the nasal mucosa and paranasal sinuses over 6 weeks to 3 months. All patients underwent surgical debridement of sinuses by ENT surgeon. In two patients exenteration of involved eyeball done by Eye surgeons.

Neurosurgical intervention done in all 10 patients. In 8 patients craniotomy done and fungal abscess along with capsule removed, in 2 patients fungal abscess aspirated through endoscopic approach to Basi frontal lobe through cribriform plates. None of them developed post-op neuro-deficit (Figure 4A and 4B).

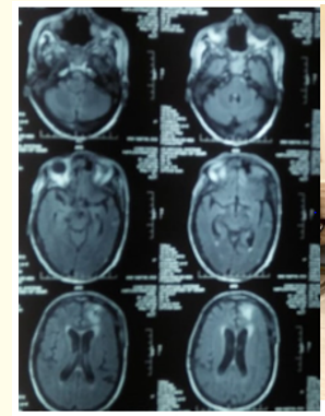

(A)

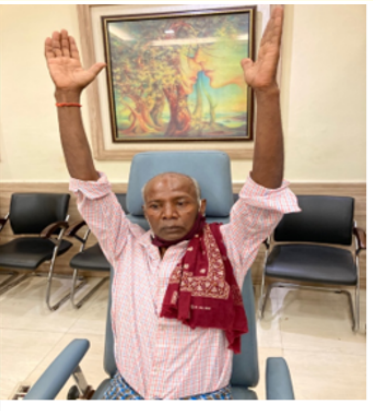

(B)
Figure 4: A. Showing postop MRI B. Showing patient came for review at 12 weeks.

The overall mortality rate was 20 per cent ( 2 of 10 ). The age of one deceased patient was 75 years, and patient died on third postop day due to uncontrolled diabetic ketoacidosis and electrolytes imbalance, the second patients age was 46 years, postoperatively patient recovered and discharged, but on $3^{\text {rd }}$ week patient died at home. In surviving patients, the follow-up period ranged from 4 to 12 weeks.

\section{Discussion}

Mucor-mycosis usually develops in severely immunocompromised patients, e.g., those with diabetes mellitus, diabetic ketoacidosis and post-transplant patients, haematological malignancy, and iatrogenic immunosuppression $[2-4,8]$. After the $2^{\text {nd }}$ phase 
of covid infections, hospitals flooded with rhino cerebral Mucor mycosis. We too faced many cases of post covid mucor-mycosis specially those treated with steroids and inhalational oxygen. The disease usually invades the nose probably through contaminated oxygen and spreads by direct extension and intravascular propagation to involve the paranasal sinuses, orbit, cribriform plate, meninges, and brain.

The patients presented with facial oedema, impaired vision, and proptosis as the most common complaints [9-11]. Orbital symptoms include loss of function of the second, third, fourth and sixth cranial nerves, with resultant ptosis, proptosis, chemosis, orbital pain, mydriasis, and loss of vision due to cavernous sinus thrombosis [12-14]. Nasal endoscopy is best for the early diagnosis in the high risk and all symptomatic patients. Endoscopies detect any changes in mucosal colour (i.e., white, grey, or black) and/or texture (i.e., granulation or ulceration) [9] for early diagnosis. It can also present with decreased mucosal bleeding or sensation $[13,15]$. Eighty per cent of cases presented with necrotic nasal mucosa during the disease in one study [16].

The cranial nerves, afferent pupillary reflex, visual acuity, and facial sensation must be evaluated. Facial oedema associated with rhino cerebral Mucor mycosis may be confused with periorbital cellulitis; however, the periorbital oedema associated with rhino cerebral Mucor mycosis is soft, cool and nontender, while the oedema of cellulitis is firm, warm, and tender. Rhino cerebral Mucor mycosis produces a paralytic ptosis: the examiner can raise the eyelid easily, but the oedematous ptosis of cellulitis resists active lid opening [13].

Unilateral gangrene and perforation of the hard and soft palates may be found due to involvement of the palatine arteries on examination of oral cavity and oropharynx [15,17].

The diagnosis of rhino cerebral Mucor mycosis is best confirmed by histological evaluation of infected tissue. Infection usually begins along the middle or inferior turbinate [18]. Biopsies can be taken from commonly involved areas such as the middle turbinate even in the absence of marked mucosal abnormality [15]. Most of the patients presented in this study had evidence of necrosis on the middle or inferior turbinate.

Histologically, the fungi are characterised by broad, thick-walled, non-septate hyphae that branch at right angles. There is a distinct proclivity for vessel wall invasion, which causes thrombosis with resultant ischaemia and infarction [17]. In contrast to most fungi, the aetiological agents of Mucor mycosis are readily seen on $\mathrm{H}$ and E-stained tissue sections [18]. Due to the possibility of contamination, the diagnosis should be verified histologically rather than by positive microbial cultures $[7,19]$.

Waiting for cultures may also delay the initiation of treatment. If a clinical picture of Mucor mycosis exists, positive direct smears may be sufficient to justify treatment [14].

Computed tomography (CT) and MRI scans may be suggestive of invasive Mucor mycosis, but they are often nondiagnostic. The most common CT findings include mild mucosal sinus thickening and extraocular muscle thickening. Later findings may include frank abscess and sinus opacification (often without air-fluid levels), together with extension into the orbit, orbital apex, or brain. If angio-invasion is present, bone erosion may be absent even in the presence of progressive disease. Magnetic resonance imaging evidence of sinus involvement may range from hyperintensity on T2weighted scans to marked hypo intensity on all sequences, often with obliteration of normal fat planes. The T2-weighted modality is typically more useful than T1-weighted scans for the evaluation of extension into the orbital apex, cavernous sinus, or brain. Computed tomography is considered best for the evaluation of bony involvement, while MRI is best for evaluating the cranial nerves and cavernous sinuses [20-22]. Overall, CT and MRI are most useful in surgical planning rather than for the diagnosis of rhino cerebral Mucor mycosis.

During our observations, CT scans showed nonspecific sinusitis findings unilaterally in six patients and bilaterally in two. In MRI, seven patients had Basi frontal cerebral Mucor mycosis while three had Basi temporal Mucor mycosis. One more interesting finding were that all female patients showed right cerebral involvement while all males showed left sided involvement.

The mainstays of rhino cerebral Mucor mycosis therapy are reversal of the cause of immunosuppression, high dose systemic amphotericin B and surgical debridement of nonviable tissue $[2,18,23,24]$.

The predisposition of diabetes mellitus patients to rhino cerebral Mucor mycosis is probably related to hyperglycaemia and acidosis. The acidotic environment is believed to promote both phagocytic dysfunction and a decrease in the iron-binding capacity of the blood. In previous reports, up to 60 - 80 per cent of patients 
with Mucor mycosis had diabetes mellitus, and one-third to onehalf of these diabetic patients were in diabetic ketoacidosis at the time of infection $[19,25]$. Recent studies have found a decreasing incidence of diabetic ketoacidosis because the characteristic signs and symptoms of the disease are now well recognised by physicians. Patients with underlying diseases that take longer to correct are known to have a lower survival rate for Mucor mycosis [10].

During our observations, out of 10 patients 5 were diagnosed case of DM before covid infection and were on oral hypoglycaemics, while 2 patients developed uncontrolled sugar due to steroid during covid treatment and 1 patient remained euglycemic. They all were treated with inhalation oxygen. The exact pathophysiology of Mucor mycosis in these post covid patients are still subject of debate and need more research.

Upon diagnosis of rhino cerebral Mucor mycosis, high-dose amphotericin B therapy should be instituted immediately. Because of the potential for nephrotoxicity, patients' renal function must be closely monitored. Liposomal preparations of amphotericin B may allow the use of this antifungal agent while reducing systemic toxicity $[18,24]$. In general, liposomal amphotericin B is reserved for clinically proven fungal infection in an immunocompromised patient with an elevated serum creatinine concentration (greater than $2.5 \mathrm{mg} / \mathrm{dl}$ ), or progression of fungal disease while receiving the maximal dosage of standard amphotericin B $(1.25 \mathrm{mg} / \mathrm{kg} /$ day $)$ [15]. Liposomal amphotericin B is safer but more expensive than conventional amphotericin B.

More extensive surgical treatment may include sinus surgery, wide resection of necrotic soft tissue and bone, and exenteration of the orbit if needed [26]. The orbit is the portal of entry for infection of the central nervous system. The most difficult decision in the management of orbital Mucor mycosis is whether to undertake orbital exenteration. In general, the indications for orbital exenteration are ophthalmoplegia, proptosis, cranial nerve involvement, cranial involvement, and ocular involvement (e.g., central retinal artery occlusion) $[10,14,17,23]$. However, several reports have documented patient survival without exenteration, in the presence of clinical orbital involvement [27-29].

Aggressive surgical debridement to remove disease outside the Sino nasal cavity rarely attains negative margins or improves long-term survival $[9,11]$. Patients with intracranial spread are less likely to respond to radical surgery, and their prognosis is extremely poor $[11,30]$. When radical surgery is considered, such patients should be appropriately counselled [15].
Irregular amphotericin B administration due to unavailability/ toxicity/intolerance high cost of treatment was major challenges during the treatment. All patients discharged with Tablet Posaconazole 100 mg 6 - 8 hourly for 6 weeks to 3 months. Doses were tapered depending on the endoscopic and MRI findings in follow up of patients.

\section{Conclusion}

Mucor mycosis is a serious disease and may be fatal if not treated in time. A high index of clinical suspicion, early diagnosis and immediate correction of underlying medical disorders are important factors for a favourable prognosis. The prognosis is good in patients with rhino-cerebral mucor-mycosis that has spread outside the Sino nasal cavity and localized in brain with aggressive medical and surgical therapy. Surgery should always be considered in those who fit in criteria as there is good survival if done early and adequately following the microsurgical principles.

\begin{tabular}{|c|c|c|c|c|c|}
\hline S.no. & $\begin{array}{c}\text { Age/ } \\
\text { sex }\end{array}$ & $\begin{array}{c}\text { Previous } \\
\text { Covid } \\
\text { history } \\
\text { and } \\
\text { treatment }\end{array}$ & $\begin{array}{l}\text { CT/MRI } \\
\text { findings }\end{array}$ & $\begin{array}{c}\text { Neurosurgical } \\
\text { approach }\end{array}$ & $\begin{array}{c}\text { Outcomes } \\
\text { after } 6 \\
\text { weeks }\end{array}$ \\
\hline 1 & $60 / \mathrm{M}$ & yes & $\begin{array}{c}\text { Left Basi } \\
\text { frontal fungal } \\
\text { abscess }\end{array}$ & Craniotomy & Recovered \\
\hline 2 & $68 / \mathrm{F}$ & yes & $\begin{array}{c}\text { Right Basi } \\
\text { frontal abscess }\end{array}$ & Endoscopic & Recovered \\
\hline 3 & $30 / \mathrm{M}$ & yes & $\begin{array}{l}\text { Left temporal } \\
\text { abscess }\end{array}$ & craniotomy & Recovered \\
\hline 4 & $75 / \mathrm{M}$ & yes & $\begin{array}{c}\text { Left Basi } \\
\text { frontal abscess }\end{array}$ & Endoscopic & $\begin{array}{c}\text { Died } 3^{\text {rd }} \\
\text { day }\end{array}$ \\
\hline 7 & $75 / \mathrm{M}$ & yes & $\begin{array}{c}\text { Left Basi } \\
\text { frontal abscess }\end{array}$ & craniotomy & Recovered \\
\hline 6 & $46 / F$ & Yes & $\begin{array}{c}\text { B/L Basi } \\
\text { frontal abscess } \\
(\mathrm{R}>\mathrm{L})\end{array}$ & Craniotomy & $\begin{array}{c}\text { Died } 3^{\text {rd }} \\
\text { week }\end{array}$ \\
\hline 7 & $67 / F$ & Yes & $\begin{array}{c}\text { Right temporal } \\
\text { abscess }\end{array}$ & craniotomy & Recovered \\
\hline 8 & $52 / \mathrm{M}$ & Yes & $\begin{array}{l}\text { Left temporal } \\
\text { abscess }\end{array}$ & craniotomy & Recovered \\
\hline 9 & $45 / \mathrm{M}$ & Yes & $\begin{array}{c}\text { Right Basi } \\
\text { frontal Abscess }\end{array}$ & craniotomy & Recovered \\
\hline 10 & $54 / \mathrm{M}$ & Yes & $\begin{array}{c}\text { Right } \\
\text { Temporal }\end{array}$ & Craniotomy & Recovered \\
\hline
\end{tabular}

Table 1: Cases in which Neurosurgical intervention was done. 


\section{Bibliography}

1. Yohai RA., et al. "Survival factors in rhino-orbital-cerebral $\mathrm{Mu}$ cor mycosis". Survey of Ophthalmology 39 (1994): 3-22.

2. Mohindra S., et al. "Rhino cerebral Mucor mycosis: the disease spectrum in 27 patients”. Mycoses 50 (2007): 290-296.

3. Sundaram C., et al. "Cerebral zygomycosis”. Mycoses 48 (2005): 396-407.

4. Yeung CK., et al. "Invasive disease due to mucorales: a case report and review of the literature". Hong Kong Medical Journal 7 (2001): 180-188.

5. Luna JD., et al. "Intraconal amphotericin B for the treatment of rhino-orbital Mucor mycosis". Ophthalmic Surgery, Lasers, and Imaging Retina 27 (1996): 706-708.

6. Blitzer AX., et al. "Patient survival factors in paranasal sinus Mucor mycosis”. Laryngoscope 90 (1980): 635-648.

7. Peterson KL., et al. "Rhino cerebral Mucor mycosis: evolution of the disease and treatment options". Laryngoscope 107 (1997): 855-862.

8. Sims CR and Zeichner LO. "Contemporary treatment and outcomes of zygomycosis in a non-oncologic tertiary care center". Archives of Medical Research 38 (2007): 90-93.

9. Gillespie MB., et al. "An approach to fulminant invasive fungal rhinosinusitis in the immunocompromised host". Archives of Otolaryngology-Head and Neck Surgery 124 (1998): 520-526.

10. Hargrove RN., et al. "Indications for orbital exenteration in $\mathrm{Mu}$ cor mycosis". Ophthalmic Plastic and Reconstructive Surgery 22 (2006): 286-291.

11. Turunc T., et al. "Eleven cases of Mucor mycosis with atypical clinical manifestations in diabetic patients". Diabetes Research and Clinical Practice 82 (2008): 203-208.

12. Pillsbury HC and Fischer ND. "Rhino cerebral Mucor mycosis". Archives of Otolaryngology 103 (1997): 600-604.

13. Bodenstein NP., et al. "Clinical signs of orbital ischemia in rhino-orbital cerebral Mucor mycosis”. Laryngoscope 103 (1993): 1357-1361.

14. Talmi YP., et al. "Rhino-orbital and rhino-orbit cerebral mucormycotic". Otolaryngology-Head and Neck Surgery 127 (2002): 22-31.
15. Gillespie MB and O'Malley BW. "An algorithmic approach to the diagnosis and management of invasive fungal rhinosinusitis in the immunocompromised patient". Otolaryngologic Clinics of North America 33 (2000): 323-334.

16. Gravesen S. "Fungi as a cause of allergic disease". Allergy 34 (1979): 135-154.

17. Alleyne CH., et al. "Long term survival of a patient with invasive cranial base rhino cerebral Mucor mycosis treated with combined endovascular, surgical, and medical therapies: case report". Neurosurgery 45 (1999): 1461-1464.

18. Ferguson BJ. "Mucor mycosis of the nose and paranasal sinuses". Otolaryngologic Clinics of North America 3 (2000): 349365.

19. Jung SH., et al. "Rhino cerebral Mucor mycosis: consideration of prognostic factors and treatment modality". Auris Nasus Larynx 36 (2009): 274-279.

20. Spellberg B., et al. "Novel perspectives on Mucor mycosis: pathophysiology, presentation, and management". Clinical Microbiology Reviews 18 (2005): 556-569.

21. Centeno RS., et al. "CT scanning in rhino cerebral Mucor mycosis and aspergillosis". Radiology 14 (1981): 383-389.

22. Terk MR., et al. "MR imaging in rhinocerebral and intracranial mucormycosis with CT and pathologic correlation". Magnetic Resonance Imaging 10 (1992): 81-87.

23. Weir NW and Golding-Wood DG. "Infective rhinitis and sinusitis”. In: editions. Scott-Brown's Otolaryngology, $6^{\text {th }}$ edition. Oxford: Butterworth-Heinemann 4.8 (1997): 1-4.

24. Avet., et al. "Endoscopic sinus surgery in the management of Mucor mycosis". Journal of Neuro-Ophthalmology 199919 (1999): 56-61.

25. Bonifaz A., et al. "Palatal zygomycosis: experience of 21 cases". Oral Diseases 14 (2008): 569-574.

26. Scheckenbach K., et al. "Emerging therapeutic options in fulminant invasive rhino cerebral Mucor mycosis". Auris Nasus Larynx 37 (2010): 322-328.

27. Hejny C., et al. "Rhino-orbital Mucor mycosis in a patient with acquired immunodeficiency syndrome (AIDS) and neutropenia". American Journal of Ophthalmology 132 (2001): 111-112. 
28. Tarani L., et al. "Long-term Posaconazole treatment and follow-up of rhino-orbital-cerebral Mucor mycosis in a diabetic girl". Pediatric Diabetes 10 (2009): 289-293.

29. Kohn R and Hepler R. "Management of limited rhino-orbital Mucor mycosis without exenteration". Ophthalmology 92 (1985): 1440-1404.

30. Anaissie EJ and Shikhani AH. "Rhino cerebral Mucor mycosis with internal carotid occlusion: report of two cases and review of the literature". Laryngoscope 95 (1985): 1107-1113.

Volume 3 Issue 12 December 2021

(c) All rights are reserved by Brajesh Kumar., et al. 